\title{
A significant feature of microvessels in magnifying narrow-band imaging for diagnosis of early gastric cancer
}

Authors

Institutions
Takashi Kanesaka', Noriya Uedo', Kenshi Yao² ${ }^{2}$ Yasumasa Ezoe ${ }^{3}$, Hisashi Doyama ${ }^{4}$, Ichiro Oda ${ }^{5}$, Kazuhiro Kaneko $^{6}$, Yoshiro Kawahara ${ }^{7}$, Chizu Yokoi ${ }^{8}$, Yasushi Sugiura ${ }^{9}$, Hideki Ishikawa ${ }^{10}$, Minoru Kato ${ }^{1}$, Yoji Takeuchi', Manabu Muto ${ }^{3}$, Yutaka Saito

Institutions are listed at the end of article. submitted 6. March 2015 accepted after revision 16. June 2015

\section{Bibliography}

Dol http://dx.doi.org/

10.1055/s-0034-1392608

Published online: 2.9.2015

Endoscopy International Open 2015; 03: E590-E596

(c) Georg Thieme Verlag KG Stuttgart · New York

E-ISSN 2196-9736

\section{Corresponding author}

\section{Noriya Uedo, MD}

Department of Gastrointestinal Oncology

Osaka Medical Center for Cancer and Cardiovascular Diseases

1-3-3 Nakamichi Higashinari-ku Osaka 537-8511

Japan

Fax: +81-6-69814067

uedou-no@mc.pref.osaka.jp
Background and study aims: Magnifying narrowband imaging (NBI) is more useful than conventional endoscopy for diagnosing early gastric cancer (EGC). However, evaluation of irregular microvascular patterns is subjective and is often difficult, even with expert eyes. The aim of this study was to clarify the most important microvascular patterns in magnifying NBI for diagnosis of EGC. Patients and methods: This was a post-hoc analysis of a multicenter prospective trial among nine Japanese hospitals. A total of 1353 patients underwent screening with white-light endoscopy and 362 patients had small $(\leq 10 \mathrm{~mm})$ depressed lesions. They were randomly assigned to magnifying NBI or white-light endoscopy followed by magnifying NBI. During diagnosis, magnifying NBI images were recorded before biopsy. All magnifying NBI images were reviewed and evaluated for the association of four features of microvessels - that is, dilation, tortuosity, difference in caliber, and variation in shape - with cancer diagnosis.

\section{Introduction}

\section{$\nabla$}

Early diagnosis by endoscopy is the first step for curative treatment of gastric cancer [1]. Early gastric cancer (EGC) without risk of lymph node metastasis can be removed curatively by endoscopic resection, which is a minimally invasive treatment $[2,3]$. However, accurate diagnosis for EGC using conventional white-light endoscopy (WLE) is usually difficult because most EGCs show only subtle macroscopic findings.

Narrow-band imaging (NBI) is an opticodigital image-enhanced technology [4]. In combination with magnifying endoscopy, NBI can clearly visualize the microsurface structure and microvascular architecture of the gastric mucosa that corresponds with histological findings [5]. Yao et al. indicated that irregular microvascular patterns and demarcation lines are diagnostic criteria for EGC with magnifying NBI [6-8]. In a multicenter
Results: Images of 343 lesions (40 cancerous and 303 benign depression lesions) were evaluable. The diagnostic performance (sensitivity/specificity) of each finding was: dilation, 25/90\%; tortuosity, 55/24\%; difference in caliber, $13 / 99 \%$; and variation in shape, $70 / 95 \%$. Multivariate analysis identified only variation in shape as being statistically significantly associated with diagnosis of cancer (odds ratio 38.0, 95\% confidence interval: $16.1-95.7, P<0.001)$. All findings showed moderate agreement ( $\mathrm{K}$ values): dilation, 0.44 ; tortuosity, 0.33 ; difference in caliber, 0.26 ; and variation in shape, 0.48 .

Conclusions: A variation in shape was the most significant feature of microvessels observed in magnifying NBI for diagnosis of small depressedtype EGC.

Study registration: UMIN-CTR000001072

prospective randomized controlled trial, magnifying NBI using those criteria was more useful than conventional WLE for diagnosis of small depressed EGC during screening endoscopy [9]. Although irregular microvascular pattern is a key diagnostic criterion for EGC, evaluation of microvascular irregularities is often subjective and requires expertise.

For esophageal squamous cell carcinoma, Yoshida et al. indicated that microvascular irregularity consists of four components: dilation, tortuosity, change in caliber, and variation in shape [10], and the presence of all four components is suggestive of diagnosis of carcinoma [11]. Kaise et al. investigated the microvascular characteristics of superficial depressed-type cancer and indicated that, as well as squamous cell carcinoma, dilation, tortuosity, abrupt caliber alteration, and heterogeneity were significantly associated with diagnosis of EGC [12]. However, which microvascular 


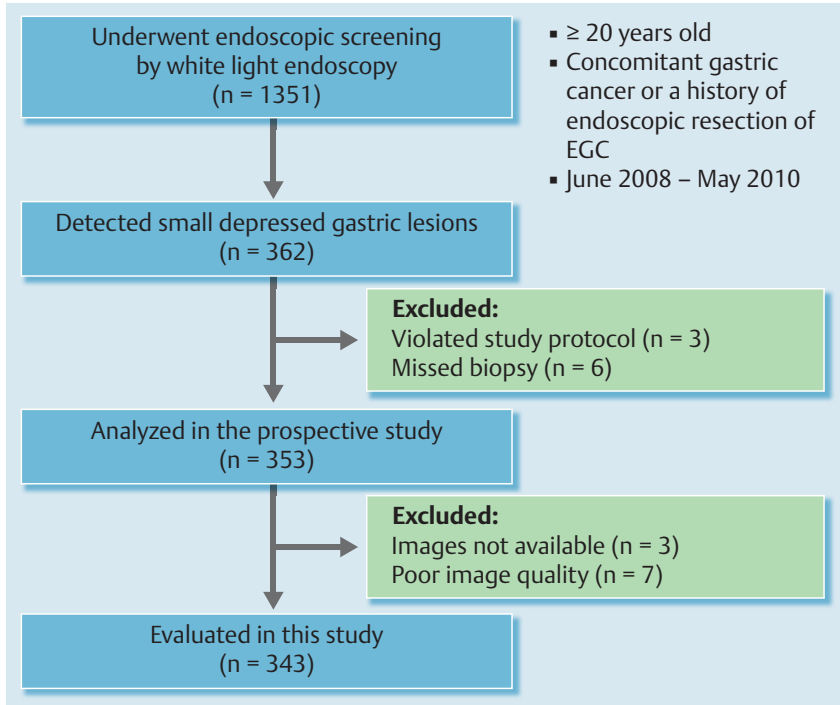

Fig. 1 Method for selection of samples in this study.

characteristics are the most important for diagnosis of EGC is unclear. Therefore, the aim of this study was to clarify the significant features of microvascular irregularities on magnifying NBI for diagnosis of EGC.

\section{Patients and methods \\ $\nabla$}

\section{Study design and participants}

This study was a post-hoc analysis of a multicenter, prospective, randomized controlled trial in nine hospitals in Japan (UMINCTR000001072) [9]. The protocol of the trial was approved by the Ethics Committee of the Kyoto University Graduate School of Medicine on 14 February 2008. The trial was conducted according to the Standards for the Reporting of Diagnostic Accuracy Studies (STARD) initiative [13] and followed the Declaration of Helsinki.

Patients aged $\geq 20$ years with concomitant gastric cancer or a history of endoscopic resection of EGC were recruited between June 2008 and May 2010. Exclusion criteria were a history of gastric resection, severe organ failure, and anticoagulant medication. A total of 1353 patients were enrolled and underwent endoscopic screening with conventional WLE. In total, 362 patients had targeted lesions that were defined as newly detected and undiagnosed small, depressed gastric lesions $\leq 10 \mathrm{~mm}$ in diameter. Only the first lesion detected in each patient was chosen for evaluation. They were randomly assigned to the WLE $(n=180)$ or magnifying NBI $(n=182)$ groups. Diagnosis of the target lesions was made on-site by a single endoscopist on the basis of predetermined diagnostic criteria for WLE and magnifying NBI, and the results were documented on case report forms. In the WLE group, magnifying NBI was performed after completion of WLE to evaluate the additional diagnostic yield of magnifying NBI over WLE. At least two endoscopic images of the targeted lesion in each mode were recorded during diagnosis and stored in a computer image server.

After accomplishing all endoscopic diagnoses, at least one biopsy specimen was obtained from the target lesion. Lesions that were diagnosed as cancer or suspicious for cancer were removed by endoscopic submucosal dissection (ESD) for final histological di-

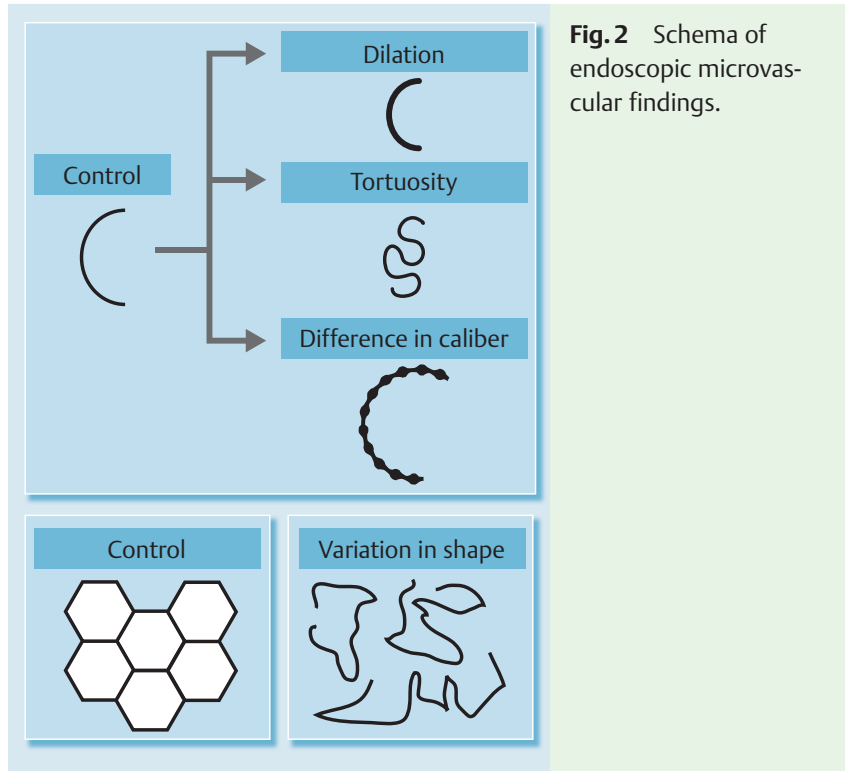

agnosis. Excluding three patients who did not follow the study protocol and six who missed biopsy, 353 patients were analyzed in the prospective trial ( $\bullet$ Fig. 1 ).

Biopsy and ESD specimens were histologically evaluated using hematoxylin and eosin staining. The histological diagnosis was made according to the revised Vienna classification [14]. Categories 4 (noninvasive, high grade neoplasia) and 5 (invasive neoplasia) were classified into cancer, and Categories 1 (negative for neoplasia), 2 (indefinite for neoplasia), and 3 (noninvasive, low grade neoplasia) were diagnosed as non-cancer. When the diagnosis was not determined at regional hospitals, the pathological slides were sent to a consultant pathologist for central review and final diagnosis. Study samples comprised 40 cancerous and 313 noncancerous lesions.

\section{Endoscopy system and setting}

The endoscopy system used in the prospective trial consisted of a video processor (CV-260SL; Olympus Medical Systems, Tokyo, Japan) and a light source (CLV-260SL; Olympus Medical Systems) that worked in both the high resolution WLE and NBI modes. NBI uses narrow-band short-wavelength light (400-430 and $525-555 \mathrm{~nm}$ ) to contrast vascular architecture and surface structure of the superficial mucosa [4]. Zoom videogastroscopy that can achieve a maximum of 80 -fold optical magnification was used (GIF-Q240Z, GIF-H260Z and GIF-FQ260Z; Olympus Medical Systems). A soft black hood (MB162 or MB46; Olympus Medical Systems) was attached to the tip of the endoscope. The structure enhancement of the endoscopic video processor was set to Bmode level 4 or 6 for WLE and to B-mode level 8 for magnifying NBI. The color mode was set to level 1 .

\section{Evaluation of microvascular findings}

We collected magnifying NBI images of all targeted lesions from each participating hospital. An experienced endoscopist (T.K.), who had experience with magnifying NBI diagnosis in $>1000$ cases, reviewed the recorded endoscopic images in magnifying NBI and evaluated the presence or absence of each of the following microvascular features in the target lesions ( $\bullet$ Fig.2).(1) Dilation: presence of a group of microvessels whose calibers were $\geq 1.5$ times as wide as those of the reference microvessels ( $\bullet$ Fig. $\mathbf{3 a}$ ). (2) Tortuosity: presence of a group of microvessels that were 

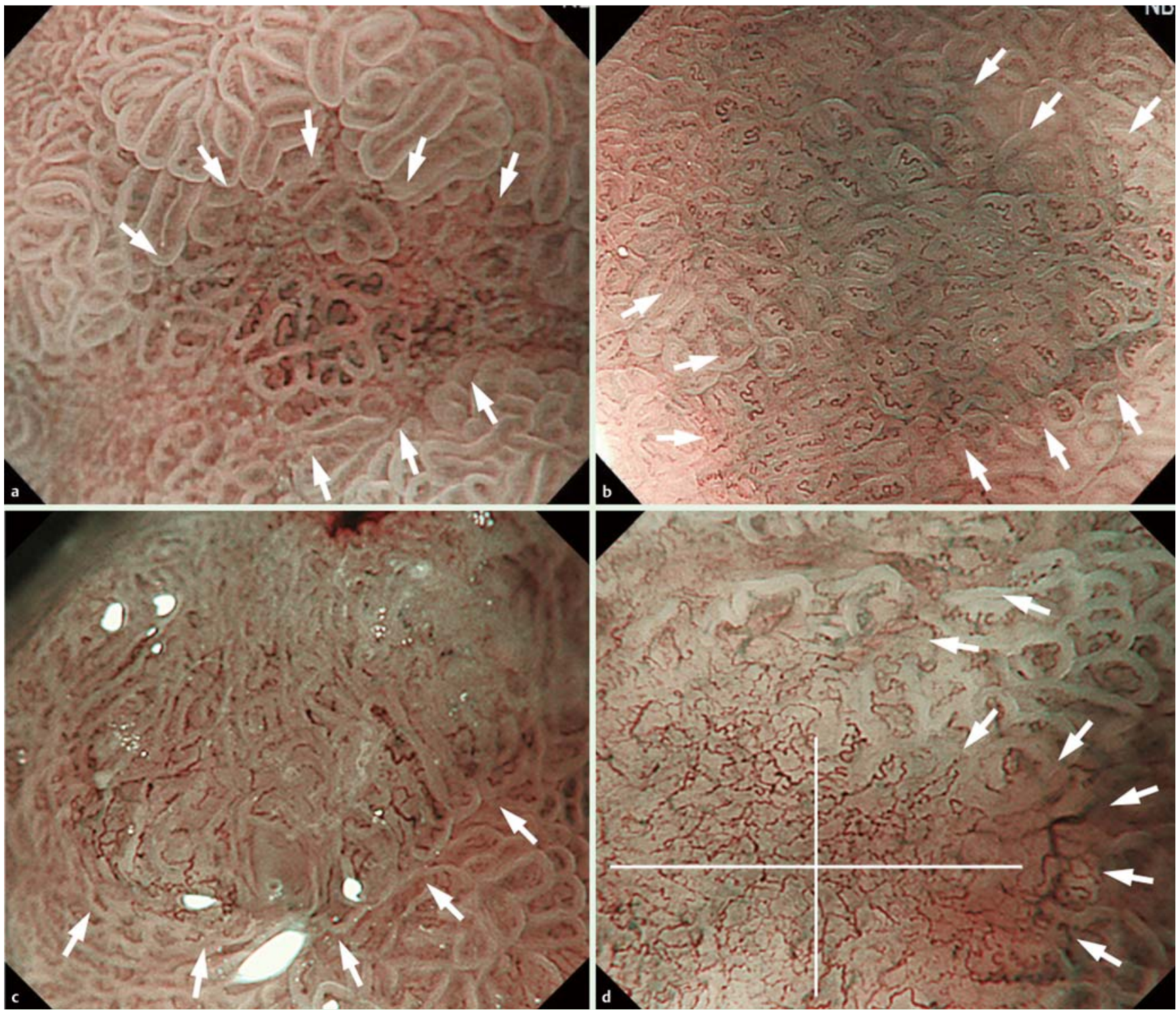

Fig.3 Representative cases for each endoscopic microvascular finding. Target lesions indicated with white arrows. a Case 1: dilation and tortuosity were present, but difference in caliber and variation in shape were absent. This lesion was histologically diagnosed as noncancerous. b Case 2: tortuosity was present but dilation, difference in caliber, and variation in shape were absent. This lesion was histologically diagnosed as noncancerous. c Case 3: dilation, difference in caliber and variation in shape were present, but tortuosity was absent. This lesion was histologically diagnosed as cancerous. $\mathbf{d}$ Case 4 : tortuosity and variation in shape were present but dilation and difference in caliber were absent. This lesion was histologically diagnosed as cancerous.

twisted or bent ( $\bullet$ Fig. $\mathbf{3 b}$ ). (3) Difference in caliber: presence of a group of microvessels whose calibers abruptly became less than half or more than double their original size ( $\bullet$ Fig.3c). (4) Variation in shape: presence of a group of microvessels whose shape and size were highly variable $(\bullet$ Fig. $\mathbf{3 d})$. In some cases, microvascular networks formed polygonal loops whose shape and size were highly variable.

As a reference for microvascular thickness and irregularity, endoscopic images of normal corpus and antral mucosa were prepared ( $\mathbf{F i g . 4} \mathbf{4 a}, \mathbf{b})$. To assess inter-observer agreement for the microvascular findings, another experienced endoscopist (N. U., experience with magnifying NBI diagnosis in $>1000$ cases) and a less-experienced endoscopist (M. K., experience with magnifying NBI diagnosis in $<100$ cases) were invited. Interpretation of the microvascular findings was discussed between the three endoscopists using other sets of magnifying NBI images to merge and ensure quality of diagnosis before starting evaluation.

\section{Statistical analysis}

For diagnostic performance, sensitivity, specificity, positive predictive value (PPV), negative predictive value (NPV), and accuracy were calculated. The $\mathrm{x}^{2}$ test, Fisher's exact test and Mann-Whitney $U$ test were used to compare demographics between cancerous and noncancerous groups. Multivariate logistic regression analysis was used to evaluate the association of each microvascular finding with cancer diagnosis. $P<0.05$ was considered statistically significant. $\mathrm{K}$ values were calculated for inter-observer agreement. Agreement was classified as excellent for $\geq 0.8$; good for $<0.8$ to $\geq 0.6$; moderate for $<0.6$ to $\geq 0.4$; and fair for $<0.4$. Statistical analyses were performed using JMP version 9 (SAS Institute, Cary, NC, USA). 

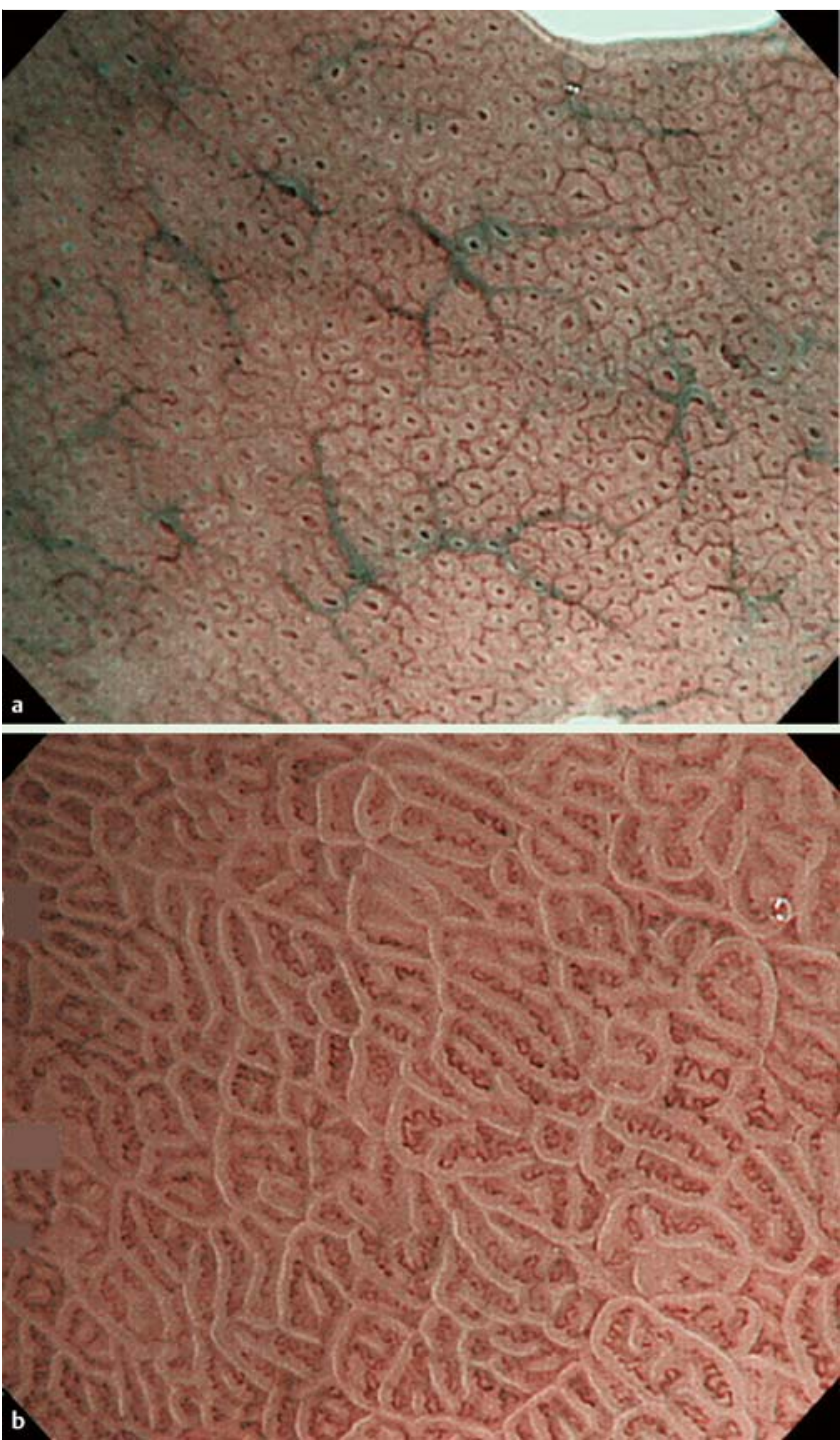

Fig.4 Images of normal gastric mucosa as microvascular control. Subepithelial capillaries of normal corpus (a) and antral (b) mucosa served as microvascular controls.

\section{Results}

$\nabla$

\section{Participants}

Among 353 patients who were analyzed in the prospective trial, endoscopic images were not available for three and appropriate images were not recorded for seven. Consequently, 343 lesions (40 cancerous and 303 noncancerous lesions) were analyzed in the present study $(\bullet$ Fig. 1 ). The patient demographics are summarized in $\bullet$ Table 1 . The cancers consisted of 39 differentiated type and one undifferentiated type.

\section{Diagnostic performance of each microvascular finding for EGC}

The diagnostic performance of the microvascular findings is summarized in $\bullet$ Table 2. Variation in shape showed good sensitivity (70\%) and high specificity (95\%) for diagnosis of EGC. Dilation and difference in caliber were specific findings (90\% and $99 \%$, respectively) but they were found in $\leq 25 \%$ of cases (sensitivity of $25 \%$ and $13 \%$, respectively). Sensitivity and specificity of tortuosity were not satisfactory (55\% and $24 \%$, respectively). Overall

\begin{tabular}{|c|c|c|}
\hline & $\begin{array}{l}\text { Cancerous } \\
\text { lesion } \\
(n=40)\end{array}$ & $\begin{array}{l}\text { Noncancerous } \\
\text { lesion } \\
(n=303)\end{array}$ \\
\hline Median age (range), years & $72(55-85)$ & $68(37-93)$ \\
\hline \multicolumn{3}{|l|}{ Gender } \\
\hline Male & 34 & 237 \\
\hline Female & 6 & 66 \\
\hline \multicolumn{3}{|l|}{ Endoscope } \\
\hline GIF-Q240Z & 15 & 117 \\
\hline GIF-H260Z & 23 & 185 \\
\hline GIF-FQ260Z & 2 & 1 \\
\hline Median size of lesions (range), $\mathrm{mm}$ & $7(3-10)$ & $5(2-10)$ \\
\hline \multicolumn{3}{|l|}{ Location of lesions } \\
\hline Upper third & 13 & 95 \\
\hline Middle third & 7 & 70 \\
\hline Lower third & 20 & 138 \\
\hline
\end{tabular}

accuracy was good $(\geq 80 \%)$ for dilation, difference in caliber and variation in shape, whereas that of tortuosity was low at $28 \%$.

\section{Association between microvascular findings and diagnosis of EGC}

All microvascular findings were significantly associated with diagnosis of cancerous lesions in univariate analysis ( $\bullet$ Table 3 ). Multivariate analysis identified only variation in shape as a significant independent predictor for EGC (odds ratio, 38.0; 95\% confidence interval [CI], $16.1-95.7 ; P<0.001$ ). Tortuosity showed an inverse association with diagnosis of EGC in univariate analysis, although multivariate analysis did not show statistical significance.

\section{Reproducibility of microvascular findings}

For inter-observer agreement of the aforementioned findings between experienced endoscopists ( $\mathrm{K}$ values), dilation (0.44) and variation in shape $(0.48)$ had moderate agreement but tortuosity $(0.33)$ and difference in caliber $(0.26)$ showed fair agreement $(\bullet$ Table4). The inter-observer agreement with a less-experienced endoscopist showed fair agreement for all microvascular findings, suggesting a requirement for some experience for evaluating microvasculature, but a similar tendency of $\mathrm{K}$ values was observed for each finding: dilation (0.29) and variation in shape (0.25) had better agreement than tortuosity (0.07) and difference in caliber (0.11).

\section{Discussion}

$\nabla$

A previous study on the association between magnifying NBI of microvessels and cancer diagnosis indicated that all four components of microvascular irregularity (dilation, tortuosity, difference in caliber, and variation in shape) were equally associated with diagnosis of depressed-type EGC [12]. However, in this study, we identified variation in shape as the most significant microvascular feature in magnifying NBI for diagnosis of small, depressed-type EGC. The independent significance of the variation in shape for diagnosis of small, depressed-type EGC did not change when we included the demarcation line, another diagnostic criterion for diagnosis of EGC [8,9], as a co-variable in multivariate analysis (data not shown). 
Table2 Diagnostic performance of each microvascular finding.

\begin{tabular}{|c|c|c|c|c|c|}
\hline Microvascular findings [95\% $\mathrm{Cl}], \%$ & Sensitivity & Specificity & PPV & NPV & Accuracy \\
\hline Dilation & $\begin{array}{l}25 \\
{[12-38]}\end{array}$ & $\begin{array}{l}90 \\
{[87-93]}\end{array}$ & $\begin{array}{l}26 \\
{[12-39]}\end{array}$ & $\begin{array}{l}90 \\
{[87-93]}\end{array}$ & $\begin{array}{l}83 \\
{[78-87]}\end{array}$ \\
\hline Tortuosity & $\begin{array}{l}55 \\
{[40-70]}\end{array}$ & $\begin{array}{l}24 \\
{[19-29]}\end{array}$ & $\begin{array}{l}8.7 \\
{[5.2-12]}\end{array}$ & $\begin{array}{l}80 \\
{[76-85]}\end{array}$ & $\begin{array}{l}28 \\
{[19-37]}\end{array}$ \\
\hline Difference in caliber & $\begin{array}{l}13 \\
{[2.3-23]}\end{array}$ & $\begin{array}{l}99 \\
{[98-100]}\end{array}$ & $\begin{array}{l}71 \\
{[38-100]}\end{array}$ & $\begin{array}{l}90 \\
{[86-93]}\end{array}$ & $\begin{array}{l}89 \\
{[86-93]}\end{array}$ \\
\hline Variation in shape & $\begin{array}{l}70 \\
{[56-84]}\end{array}$ & $\begin{array}{l}95 \\
{[93-98]}\end{array}$ & $\begin{array}{l}67 \\
{[52-81]}\end{array}$ & $\begin{array}{l}96 \\
{[94-98]}\end{array}$ & $\begin{array}{l}92 \\
{[90-95]}\end{array}$ \\
\hline
\end{tabular}

$\mathrm{Cl}$, confidence interval; PPV, positive predictive value; NPV, negative predictive value.

Table 3 Association of each microvascular finding with cancer.

\begin{tabular}{|c|c|c|c|c|}
\hline \multirow[t]{2}{*}{ Microvascular findings } & \multicolumn{2}{|c|}{ Univariate analysis } & \multicolumn{2}{|c|}{ Multivariate analysis } \\
\hline & $\begin{array}{l}\text { Odds ratio } \\
{[95 \% \mathrm{Cl}]}\end{array}$ & $P$ value & $\begin{array}{l}\text { Odds ratio } \\
{[95 \% \mathrm{Cl}]}\end{array}$ & $P$ value \\
\hline Dilation & $\begin{array}{l}3.1 \\
{[1.4-7.1]}\end{array}$ & 0.008 & $\begin{array}{l}1.9 \\
{[0.5-5.8]}\end{array}$ & 0.312 \\
\hline Tortuosity & $\begin{array}{l}0.4 \\
{[0.2-0.8]}\end{array}$ & 0.007 & $\begin{array}{l}0.6 \\
{[0.2-1.7]}\end{array}$ & 0.369 \\
\hline Difference in caliber & $\begin{array}{l}21.5 \\
{[4.0-115.0]}\end{array}$ & $<0.001$ & $\begin{array}{l}4.8 \\
{[0.5-63.8]}\end{array}$ & 0.200 \\
\hline Variation in shape & $\begin{array}{l}48.2 \\
{[20.3-114.2]}\end{array}$ & $<0.001$ & $\begin{array}{l}38.0 \\
{[16.1-95.7]}\end{array}$ & $<0.001$ \\
\hline
\end{tabular}

Although we used the same four findings of microvascular irregularity as in the previous study by Kaise et al. [12], they were modified to make interpretation simpler and more objective. Before starting this study, we noticed that microvessels in the surrounding mucosa were not observable in some cases ( $\bullet$ Fig. $\mathbf{3 a}$ ). Therefore, we prepared images of subepithelial capillaries in the normal gastric mucosa as a reference control, and the thickness and tortuosity of microvessels were compared with those of normal mucosa. In the previous study, microvascular findings in a lesion were compared with those of the surrounding mucosa. Accordingly, dilation did not show statistical significance in the multivariate analysis in the present study. Tortuosity was defined as unpredictably twisted or bent in the previous study [12] but we defined it simply according to geometrical change in the microvessels. Tortuosity had a positive association with EGC in the previous study [12], whereas it showed the opposite association in the present study, indicating that microvascular tortuosity was seen more frequently in the surrounding non-neoplastic mucosa than in the cancerous mucosa. The presence of unpredictably twisted or bent microvessels in the previous study [12] was included in variation in shape in our definition because these microvessels showed variety among uniformly tortuous vessels in the surrounding mucosa.

Another reason our results differed from the previous study by Kaise et al. [12] was the difference in patient demographics. We enrolled newly detected and undiagnosed lesions in consecutive high risk patients, whereas the previous study enrolled patients with known EGC lesions that were planned for removal by ESD. Our study samples were prospectively accumulated endoscopic images of consecutive patients. Moreover, image recording was required in the protocol, so 343 images (97\%) from 353 enrolled patients were analyzed in our study. We believe that our data reflect actual clinical settings.
Table 4 Inter-observer agreement for the microvascular findings.

\begin{tabular}{|c|c|c|}
\hline \multirow[t]{2}{*}{ Microvascular findings } & \multicolumn{2}{|c|}{ Inter-observer agreement (к value) } \\
\hline & $\begin{array}{l}\text { Experienced } \\
\text { endoscopist }\end{array}$ & $\begin{array}{l}\text { Less-experienced } \\
\text { endoscopist }\end{array}$ \\
\hline Dilation & 0.44 & 0.29 \\
\hline Tortuosity & 0.33 & 0.07 \\
\hline Difference in caliber & 0.26 & 0.11 \\
\hline Variation in shape & 0.48 & 0.25 \\
\hline
\end{tabular}

For diagnosis of esophageal squamous cell carcinoma, Ishihara et al. examined the association between microvascular characteristics and diagnosis of intramucosal high grade neoplasia, and suggested that brownish dot-like dilation, tortuosity, and variation in shape had a significant association [15]. Among these features, microvascular dilation had a significant independent association with diagnosis of neoplasia. Microvessels (intrapapillary capillary loop) in the normal esophageal mucosa are usually thin and straight but those in cancerous mucosa are dilated, tortuous, and heterogeneous. Esophageal squamous cell carcinoma develops from normal squamous epithelium that is not affected by chronic inflammation; therefore, those changes could be more obvious than in EGC. The gastric mucosa is normally rich in vasculature, so normal microvessels are thicker than those in squamous epithelium, making microvascular dilation in the cancerous mucosa less sensitive. Most EGC patients have chronic gastritis that alters the microvasculature of benign depressed lesions; therefore, tortuosity cannot be a specific finding in the stomach. In addition, for esophageal squamous cell carcinoma, microvascular findings in magnifying NBI are associated with depth of tumor invasion but magnifying NBI is not useful for predicting vertical invasion of EGC. The reason is that, for EGC, the invasive tissue is often not exposed at the surface and the mucosal structure 
remains, even when cancer invades the submucosa. Therefore, it is difficult to estimate reliably the depth of invasion from surface appearance [16]. Different organs can change the interpretation of microvascular findings in magnifying NBI.

Comparing the microvascular characteristics in a lesion with those in the surrounding mucosa may be a more practical way to diagnose EGC, rather than comparison with control images. Using control images for reference requires preparation of dedicated endoscopic images. However, in our experience, after training in 20-30 cases, we became familiar with the diagnosis of microvascular dilation without reference images. We always try to evaluate microvascular patterns at the maximum magnification level possible. Observation at the same maximum level contributed to objective assessment of the microvessels. Difference in caliber and variation in shape can be evaluated without reference images. Both findings showed high specificity (99\% and 95\%, respectively). To evaluate variation in shape, we usually apply imaginary crosshairs onto the region of interest; we call it a "magic cross" and it divides the lesion into four quadrants so we can compare the microvascular findings in each area.

What are the causes of these morphological microvascular changes in EGC? Microvessels observed by magnifying NBI are present in the shallow layer of the mucosa because the short wavelength light used in NBI can only penetrate the shallow layer of the mucosa [17]. In advanced cancer, cancerous glands that invade the submucosa or deeper usually have reactive interstitial fibrosis or necrotic tissue, and they are exposed on the surface but, in most intramucosal cancers, the surface is covered by the structurally atypical cancerous epithelium that has no reactive interstitial fibrosis. An immunohistochemical study with CD34 and D2-40 indicated that there was no significant angiogenesis in intramucosal gastric cancer [18]. Therefore, we speculated that the microvessels in the superficial cancerous epithelium would be deformed as a result of compression by the structurally atypical cancerous glands. Thus, the significant association of microvascular variation in shape with EGC diagnosis was reasonable. Furthermore, this may also explain the low incidence of change in caliber that was caused by cancer neoangiogenesis.

Considering the ability to generalize our results, direct application of this technology in the assessment of EGC may currently be difficult in most western countries because of inaccessibility and less experience of endoscopists with optical zoom endoscopes. High magnification observation using zoom endoscopy is definitely preferable to evaluate detailed vascular morphology such as tortuosity or caliber change, but dilation or variation in shape could be evaluated at a low magnification level. Therefore, we suggest that a combination of electronic zoom function with high definition videoendoscopy that is currently available in most western countries could be used to evaluate variation in microvascular shape. Mabe et al. indicated that an educational lecture on magnifying NBI images from an expert improved the diagnostic ability of less-experienced endoscopists [19]. Consequently, we believe that the concept advanced in this study is applicable to endoscopists in western countries after attaining certain knowledge and experience with NBI diagnosis.

This study had some limitations. First, although we used prospectively collected consecutive data with low selection bias, this was a retrospective study. Therefore, validation of diagnostic accuracy in a prospective study is required. Second, only depressed lesions with a diameter of $\leq 10 \mathrm{~mm}$ were studied; thus, it is not clear whether our results are applicable to elevated lesions. The microvascular pattern in magnifying NBI is a reliable marker for diag- nosis of depressed-type EGC but the microvasculature is sometimes not evaluable in elevated lesions. In such elevated lesions, subepithelial vessels are frequently obscured by lipid droplets in the epithelium; that is, white opaque substances that are absorbed by the neoplasm with intestinal phenotype [7]. Third, although the significant indicator of vascular finding, variation in shape, showed the highest $\mathrm{K}$ value of 0.48 , it showed a moderate level of agreement. Producing learning materials or development of an objective method to access vascular findings, such as computer-aided diagnosis systems to increase agreement, is expected.

In conclusion, variation in the shape of the microvascular pattern was the most significant feature for diagnosis of small, depressed EGC by magnifying NBI. This result facilitates interpretation of microvascular irregularity and contributes to efficient diagnosis of EGC in clinical practice.

\section{Competing interests: None}

\section{Institutions}

${ }^{1}$ Department of Gastrointestinal Oncology, Osaka Medical Center for Cancer and Cardiovascular Diseases, Osaka, Japan

${ }^{2}$ Department of Endoscopy, Fukuoka University Chikushi Hospital, Fukuoka, Japan

${ }^{3}$ Department of Therapeutic Oncology, Graduate School of Medicine, Kyoto University, Kyoto, Japan

${ }^{4}$ Department of Gastroenterology, Ishikawa Prefectural Central Hospital, Ishikawa, Japan

${ }^{5}$ Endoscopy Division, National Cancer Center Hospital, Tokyo, Japan

${ }^{6}$ Department of Gastroenterology, Endoscopy Division, National Cancer Center Hospital East, Chiba, Japan

${ }^{7}$ Division of Endoscopy, Okayama University, Okayama, Japan

${ }^{8}$ Endoscopy Division, National Center for Global Health and Medicine, Tokyo, Japan

${ }_{9}^{9}$ Division of Gastroenterology and Hepatology, Kitano Hospital, Osaka, Japan ${ }^{10}$ Department of Molecular Targeting Cancer Prevention, Kyoto Prefectural University of Medicine, Kyoto, Japan

\section{Acknowledgments \\ $\nabla$}

The study was supported in part by a Grant-in-Aid for cancer research from the Ministry of Health, Labour, and Welfare of Japan (H21-009).

Gastric NBI Study Investigators include the following: Noriya Uedo, Yoji Takeuchi, Takashi Kanesaka, Minoru Kato (Osaka Medical Cancer for Cancer and Cardiovascular Diseases); Hisashi Doyama, Yoshibumi Kaneko, Kenichi Takemura, Kazuhiro Miwa, Shinya Yamada (Ishikawa Prefectural Central Hospital); Yutaka Saito, Ichiro Oda, Shigetaka Yoshinaga, Satoru Nonaka, Shusei Fukunaga, Nobuaki Ikezawa (National Cancer Center Hospital); Manabu Muto, Yasumasa Ezoe, Shuko Morita, Takahiro Horimatsu (Kyoto University); Kenshi Yao, Takashi Nagahama, Hiroshi Tanabe, Takahiro Beppu, Yoichiro Ono, Masao Takeichi, Takao Kanemitsu (Fukuoka University Chikushi Hospital); Kazuhiro Kaneko, Tomonori Yano, Hiroaki Kon, Shinya Tsuruta (National Cancer Center Hospital East); Yoshiro Kawahara, Toshio Uraoka, Seiji Kawano, Keisuke Hori (Okayama University); Chizu Yokoi, Naoyoshi Nagata (National Center for Global Health and Medicine); Yasushi Sugiura, Atsushi Yamauchi (Kitano Hospital); Hideki Ishikawa (Kyoto Prefectural University of Medicine); and Tomoko Aoyama (Medical Research Support) 


\section{References}

1 Thrumurthy SG, Chaudry MA, Hochhauser D et al. The diagnosis and management of gastric cancer. BMJ 2013; 347: f6367

2 Isomoto $H$, Shikuwa S, Yamaguchi $N$ et al. Endoscopic submucosal dissection for early gastric cancer: a large-scale feasibility study. Gut 2009; 58: 331 - 336

3 Ono H, Kondo H, Gotoda T et al. Endoscopic mucosal resection for treatment of early gastric cancer. Gut 2001; 48: 225-229

4 Gono K, Obi T, Yamaguchi $M$ et al. Appearance of enhanced tissue features in narrow-band endoscopic imaging. J Biomed Opt 2004; 9: $568-577$

5 Muto M, Katada C, Sano Yet al. Narrow band imaging: a new diagnostic approach to visualize angiogenesis in the superficial neoplasia. Clin Gastroenterol Hepatol 2005; 3: 16-20

6 Yao K, Oishi T, Matsui T et al. Novel magnified endoscopic findings of microvascular architecture in intramucosal gastric cancer. Gastrointest Endosc 2002; 56: 279-284

7 Yao K, Iwashita A, Tanabe $\mathrm{H}$ et al. Novel zoom endoscopy technique for diagnosis of small flat gastric cancer: a prospective, blind study. Clin Gastroenterol Hepatol 2007; 5: 869-878

8 Yao K, Anagnostopoulos GK, Ragunath K. Magnifying endoscopy for diagnosing and delineating early gastric cancer. Endoscopy 2009; 41: $462-467$

9 Ezoe $Y$, Muto $M$, Uedo $N$ et al. Magnifying narrowband imaging is more accurate than conventional white-light imaging in diagnosis of gastric mucosal cancer. Gastroenterology 2011; 141: 2017-2025

10 Yoshida $T$, Inoue $H$, Usui $S$ et al. Narrow-band imaging system with magnifying endoscopy for superficial esophageal lesions. Gastrointest Endosc 2004; 59: 288-295
11 Inoue H. Magnification endoscopy in the esophagus and stomach. Dig Endosc 2001; 13: 40-S41

12 Kaise $M$, Kato $M$, Urashima $M$ et al. Magnifying endoscopy combined with narrow-band imaging for differential diagnosis of superficial depressed gastric lesions. Endoscopy 2009; 41: 310-315

13 Bossuyt PM, Reitsma JB, Bruns DE et al. The STARD statement for reporting studies of diagnostic accuracy: explanation and elaboration. Ann Intern Med 2003; 138: W1 - 12

14 Schlemper RJ, Riddell RH, Kato Y et al. The Vienna classification of gastrointestinal epithelial neoplasia. Gut 2000; 47: 251-255

15 Ishihara $R$, Inoue $T$, Uedo $N$ et al. Significance of each narrow-band imaging finding in diagnosing squamous mucosal high-grade neoplasia of the esophagus. J Gastroenterol Hepatol 2010; 25: 1410-1415

16 Uedo N, Fujishiro $M$, Goda $N$ et al. Role of narrow band imaging for diagnosis of early-stage esophagogastric cancer: current consensus of experienced endoscopists in Asia-Pacific region. Dig Endosc 2011; 23: $58-71$

17 Yao K. Zoom gastroscopy: Magnifying endoscopy in the stomach. Tokyo: Springer; 2014

18 Gao Y, Zhong WX, Mu DB et al. Distributions of angiogenesis and lymphangiogenesis in gastrointestinal intramucosal tumors. Ann Surg Oncol 2008; 15: $1117-1123$

19 Mabe K, Yao K, Nojima $M$ et al. An educational intervention to improve the endoscopist's ability to correctly diagnose small gastric lesions using magnifying endoscopy with narrow-band imaging. Ann Gastroenterol 2014; 27: 149-155 